\title{
A Rare Tumor in the Neck of a Child: Plexiform Neurofibroma
}

\author{
Case Report $\$ Siti Sarah Mohd Ramli ${ }^{1}, \oplus$ Goh Bee See ${ }^{1}$ ๑ Faizah Mohd Zaki², \\ ๑ Suria Hayati Md Pauzi ${ }^{3}$, @ Mohamad Fariz Bin Amri ${ }^{4}$ \\ ${ }^{1}$ Department of Otorhinolaryngology Head \& Neck Surgery, Pusat Perubatan University Kebangsaan Malaysia, Kuala \\ Lumpur, Malaysia \\ ${ }^{2}$ Department of Radiology, Pusat Perubatan University Kebangsaan Malaysia, Kuala Lumpur, Malaysia \\ ${ }^{3}$ Department of Pathology, Pusat Perubatan University Kebangsaan Malaysia, Kuala Lumpur, Malaysia \\ ${ }^{4}$ Department of Pathobiology and Medical Diagnostic, University Malaysia Sabah, Sabah, Malaysia
}

\section{ORCID ID of the authors:}

S.S.M.R. 0000-0001-9566-3007; G.B.S. 0000-0002-5535-2930; F.M.Z. 0000-0002-7142-3794, S.H.M.P. 0000-0001-5845-0061; M.F.B.A. 0000-0002-6803-9943.

Cite this article as: Mohd RamliSS, Bee See G, Zaki FM, Pauzi SHM, Bin Amri MF. A Rare Tumor in the Neck of a Child: Plexiform Neurofibroma. Turk Arch Otorhinolaryngol 2021; 59(4): 297-301.

Corresponding Author: Goh Bee See; irenegbs@yahoo.com

Received Date: 23.03.2021 Accepted Date: 15.08 .2021

Content of this journal is licensed under a Creative Commons Attribution 4.0 International License. Available online at www.turkarchotolaryngol.net

\section{(c) (i) $\$$}

DOI: $10.4274 /$ tao.2021.2021-3-10

Plexiform neurofibroma represents an uncommon variant of neurofibromatosis type 1 , constituting only $5 \%-30 \%$ of all cases. Plexiform neurofibroma is usually diagnosed during childhood and arises from multiple nerves, manifesting as bulging and deforming masses that can also involve connective tissue and skin folds. We report a case of a two-year-old girl who presented with worsening stridor since birth and later exhibited progressively increasing left neck swelling at the age of 10 months old. Ultrasound and magnetic resonance imaging (MRI) showed a lobulated solid mass in the left deep neck space extending to the midline and having a mass effect on the airway with involvement of the supraglottic region. Tracheostomy was done, and a biopsy of the supraglottic lesion revealed a plexiform neurofibroma. The patient was conservatively managed after a discussion with her parents concerning the associated potential of operative morbidity. The patient's parents had learned about tracheostomy care, and the patient was scheduled for yearly MRI surveillance. MRI was performed again three months after the initial diagnosis and showed stable lesion. Plexiform neurofibroma is a slow-growing tumor. A treatment decision must consider the benefits of surgery and the morbidity of the progressing disease. Hence, airway management is crucial prior to the final decision of such cases.

Keywords: Plexiform neurofibroma, neck, larynx, airway, rare diseases, tracheotomy, case report

\section{Introduction}

Plexiform neurofibroma (PN) is an uncommon variant of Neurofibromatosis type 1 , representing only $5 \%-30 \%$ of all cases (1). Neurofibromatosis type 1 (NF1 ) is a rare autosomal dominant genetic condition that occurs in 1 in 3,000-5,000 births (1). PN is the major cause of morbidity and disfigurement (2). There are few cases of PN reported in the neonatal period making the diagnosis difficult and unexpected in neonates with stridor (2). In this work, we highlighted a case of PN in a toddler who presented stridor since birth and neck swelling when the child was one year old. 


\section{Case Presentation}

A born term two-year-old Chinese girl presented with worsening noisy breathing since birth. The child had a good cry and no history of aspiration. She was treated for laryngomalacia by a clinician up to the age of six months old. The mother then noted a painless but progressively increasing swelling on the left side of the child's neck when the child was ten months old. There was no sign of inflammation. She failed to thrive, as her weight was only at the fifth percentile. Her developmental history was up to age. The child's maternal grandfather had been diagnosed with Neurofibromatosis type 1 .

Upon examination, the child was seen to have biphasic stridor with no other signs of respiratory distress. Pectus excavatum was observed. There were multiple firms, lobulated masses (levels II and III, sized $4 \times 4 \mathrm{~cm}$ ) on her left neck, with no skin changes. She had nine café-au-lait spots.

Flexible nasopharyngolaryngoscopy (FNLPS) showed a mass on the left supraglottic region that was obstructing the laryngeal inlet. The mass was also partially obscuring the view of the glottis.

An ultrasound showed a lobulated hypoechoic mass with echogenic debris within the mass (Figure 1). The mass was similar in repeated scans. Hence, magnetic resonance imaging (MRI) was performed to evaluate the lesion's character and extension. MRI showed that a lobulated heterogeneous "bag of worms" lesion occupied the left lateral neck and extended superiorly into the parapharyngeal region, posteriorly into the posterior cervical space and inferiorly up to the retrosternal and superior mediastinum. It encased the carotid vessels inferiorly. The lesion displaced the airway to the right. MRI findings were suggestive of plexiform neurofibroma with airway compromise (Figure 2).

We proceeded with a tracheostomy, direct laryngoscopy, tracheoscopy and ultrasound-guided biopsy of the neck and left arytenoid mass under general anesthesia. Direct laryngoscopy showed that the supraglottic structures had been pushed to the right by a left arytenoid mass (Figure 3). The true vocal cords, subglottis and trachea were normal.

Ophthalmology and neurology assessments were done and showed normal results. A brainstem evoked response showed normal bilateral hearing.

Histopathological assessment showed fragments of fibrocollagenous tissue composed of loosely arranged small wavy spindle cells interspersed with wisps of collagen in myxoid stroma. The spindle cells displayed uniform, round ovoid nuclei, inconspicuous nuclei, and scanty eosinophilic cytoplasm. There were few mitosis and ganglion cells, and there was no cellular atypia or necrosis, indicating the benign features of the mass (Figure 4). Immunohistochemistry showed that the spindle cells were positive for S-100 and negative for SMA and desmin (Figure 5). These findings concluded the diagnosis of plexiform neurofibroma.

Because surgery would hypothetically cause more morbidity to the patient and because it was a slow-growing tumor, she was put under yearly MRI surveillance. A year later, the patient was well, as the MRI surveillance showed no progression of the tumor.

\section{Discussion}

$\mathrm{PN}$ is a benign, slow-growing tumor which arises from superficial peripheral nerves that could be only superficial or an extension of a deeper, massive plexiform tumor $(1,3)$.

While around $25 \%-48 \%$ of PN cases are reported above the neck, symptoms are based on the sites of manifestation and present themselves early in childhood (4-6). There is a case reported in the literature of a six-year-old child who presented with emergency upper airway obstruction and was diagnosed with $\mathrm{PN}$ in a left deep parotid mass (5). In adults,
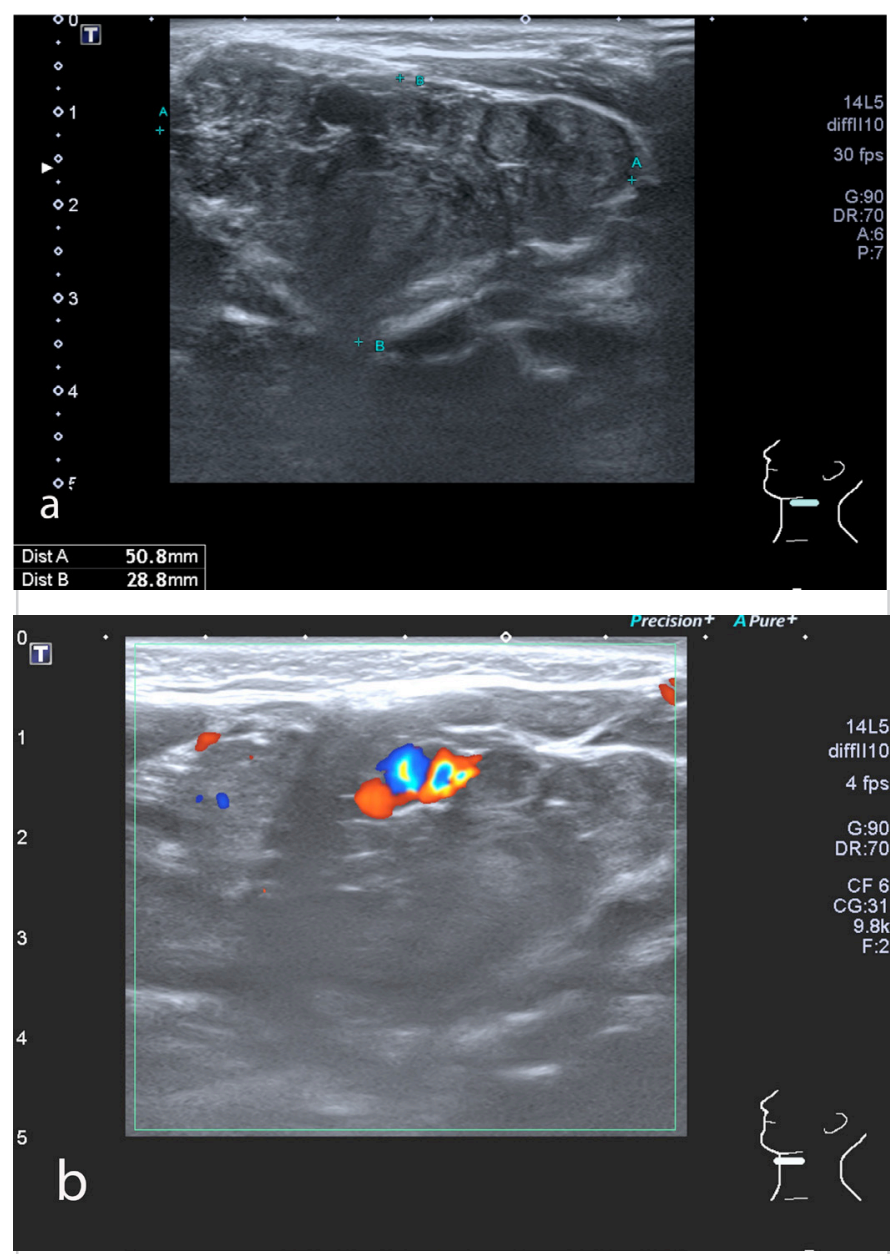

Figure 1. Ultrasound neck scan in B-mode (a) and color doppler (b) show a lobulated solid mass with internal echoes within (not compressible) and no color doppler within, respectively 

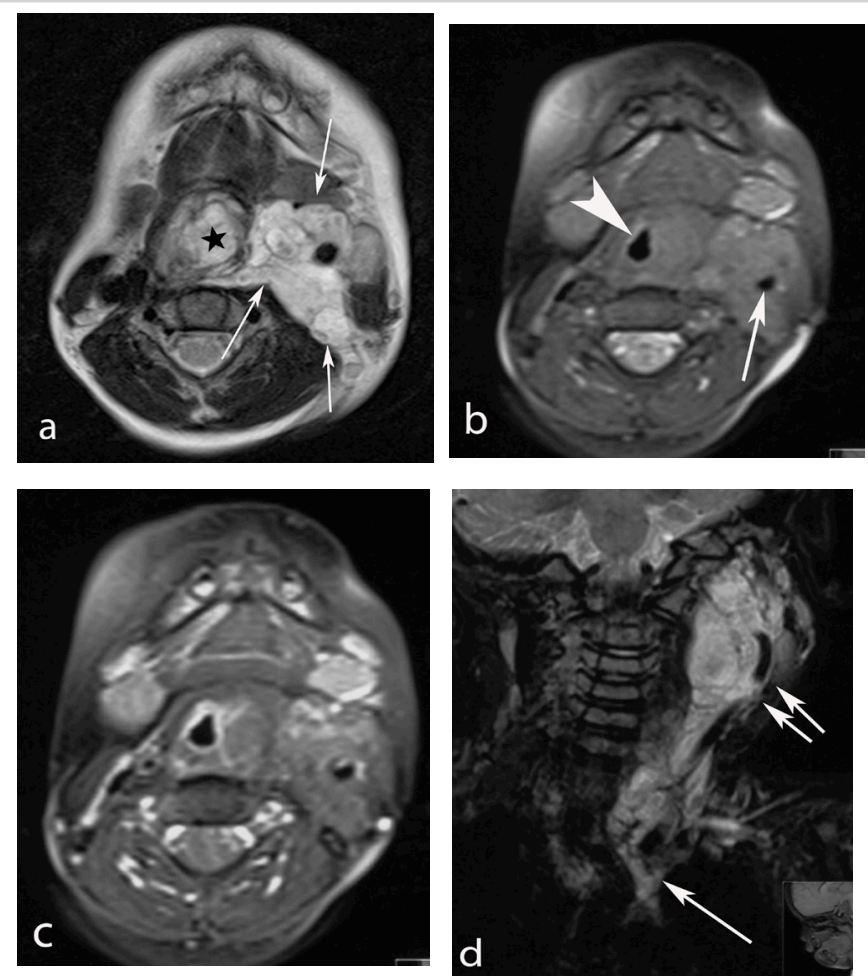

Figure 2. MRI neck scan of the patient: (a) axial T2-weighted image, (b) axial T1-weighted image, (c) axial T1-weighted postgadolinium image and (d) coronal T2 fat-saturated image. The images show a lobulated mass occupying the left lateral neck space (arrows in a and d) with medial extension to the vocal cord region, which narrows the airway (star in a and arrowhead in b). This demonstrates minimal post-gadolinium enhancement. The mass extends caudally into the region of the superior mediastinum (single arrow in d)

MRI: Magnetic resonance imaging

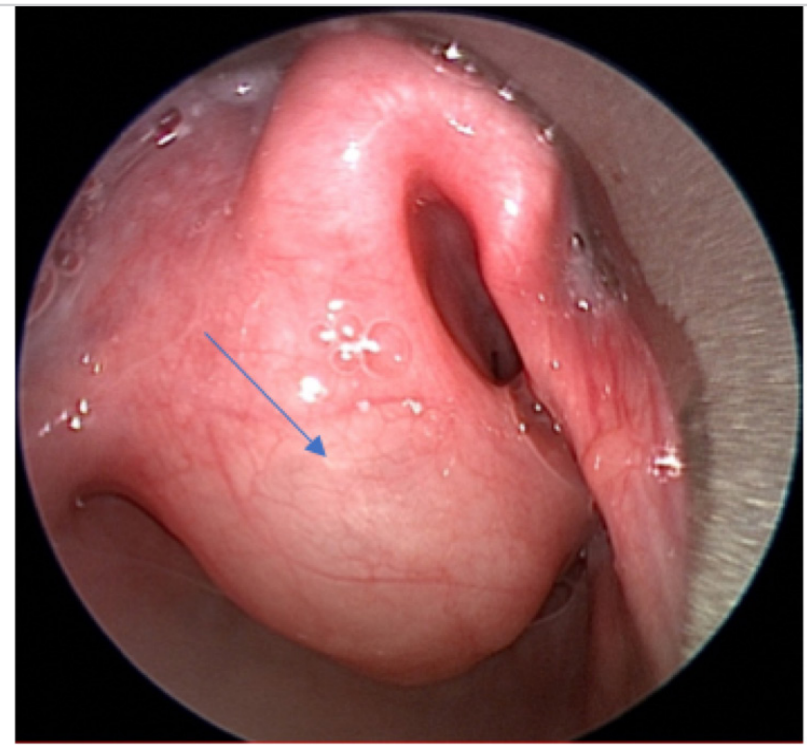

Figure 3. Picture of patient's larynx on direct laryngoscopy. Supraglottic structures were pushed to the right by a left arytenoid mass (the arrow indicates the mass)
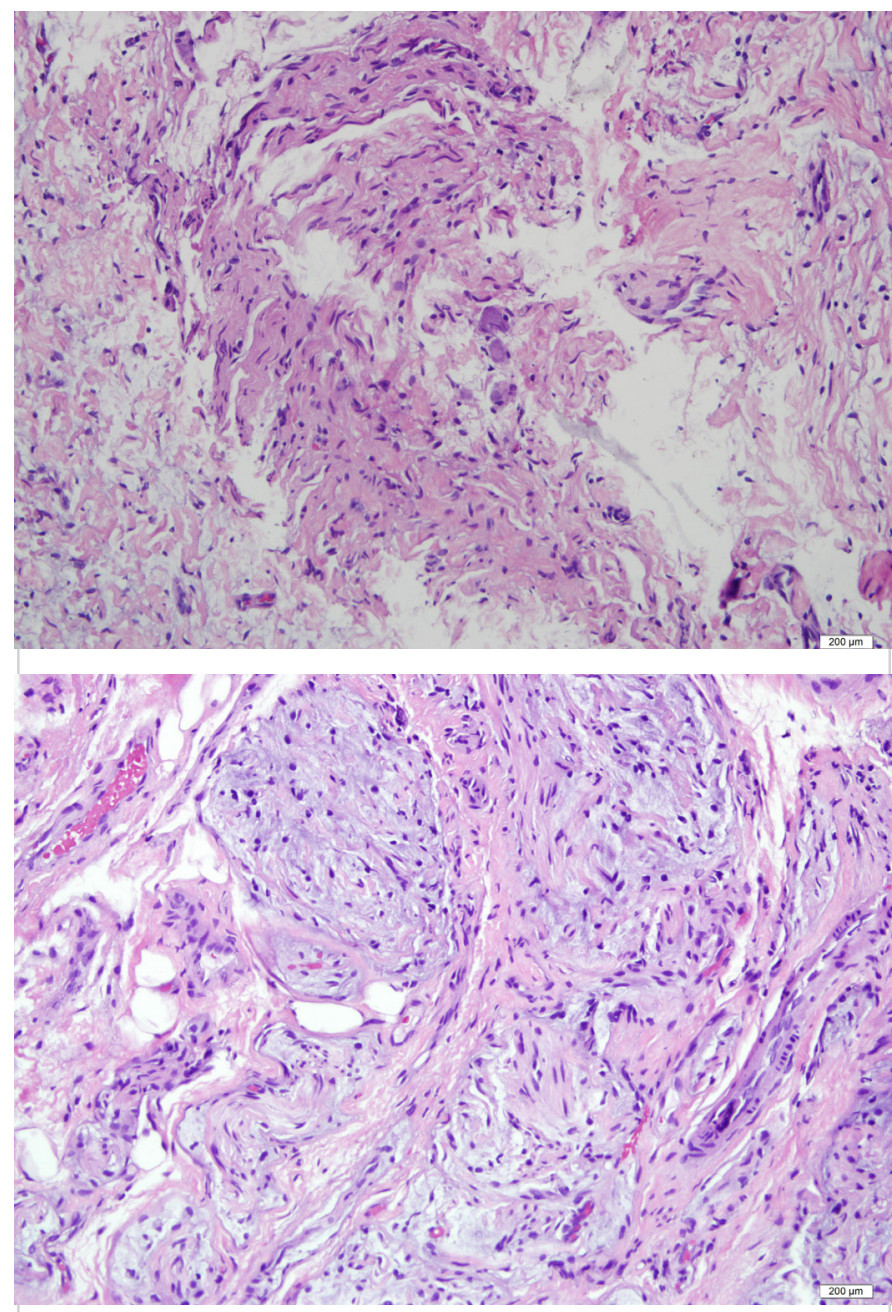

Figure 4. Histopathology of (a) the left neck mass and (b) the left arytenoid $(\times 200)$

Fragments of fibro-collagenous tissue composed of loosely arranged small, wavy spindle cells were interspersed with wisps of collagen in myxoid stroma. The spindle cells display fairly uniform, round ovoid nuclei, inconspicuous nuclei, and scanty eosinophilic cytoplasm

the main complaint is a slow-growing mass since childhood that later became infected or bothersome $(4,6)$. Patients tend to have a first degree relative diagnosed with NF-1 (1-6).

Our patient presented with stridor since birth. She was therefore treated for laryngomalacia, as it is the most common congenital cause of stridor (7). Other common causes for noisy breathing among babies are congenital subglottic stenosis, vallecular cysts, and congenital unilateral vocal fold paralysis (7). She later developed a neck mass that exacerbated the stridor symptoms and was referred to our center. We felt that the stridor symptoms would warrant earlier management and employed an endoscopic assessment to aid the early diagnosis.

MRI was used to determine the morphology, type of lesion and extent of disease (8). PN displayed high signal intensity on T2-weighted MRI, and most of the borders 

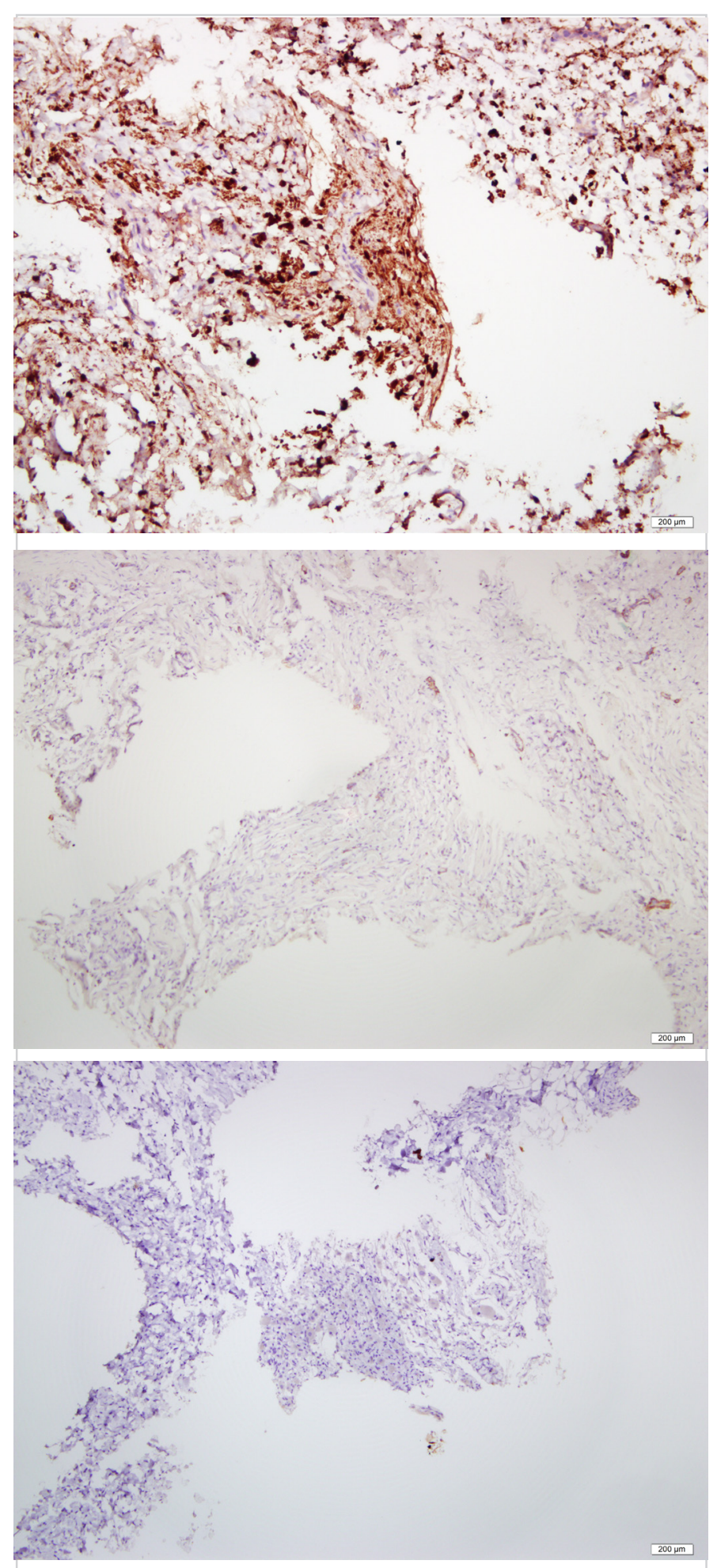

Figure 5. Immunohistochemistry of the left neck mass $(\times 100)$ (a) S-100 protein, (b) SMA, (c) Desmin

The spindle cells were positive for the $\mathrm{S}-100$ protein and negative for SMA and desmin. Hence, the diagnosis of plexiform neurofibroma was concluded.

SMA: Smooth muscle actin branched in many directions (8). The lesion was diffuse, which made it difficult to measure objectively (8). However, MRI was adequate for determining volumetric changes, and interobserver variability was less than 10\% (8).

Histopathologically, the cells in the nerve sheath proliferated along a significant length of a major nerve and multiple nerve fascicles $(3,9)$. PN was mostly immunoreactive to the S-100 protein $(3,9)$. Similar findings in the biopsy of our patient concluded the diagnosis of PN.

Surgical treatment was the main treatment for PN, as no successful cases of medical therapy have been reported in PN patients $(3,9,10)$. The decision to perform surgery is typically based on debilitating symptoms and aesthetic problems $(5,6,9,10)$. It might be possible to achieve subtotal or total resection for superficial PN that remains in the upper layer of the skin with a low morbidity risk (9). Invasive PN involving multiple tissue planes with no clear margins was impossible to resect (9). The rate of recurrence recorded for near total tumor resection was $20 \%(6,9,10)$. The rate of complications of neurological deficits in patients with head and neck PN tumors approached $10 \%(6,9,10)$. A case was reported of a 12-year-old who had facial nerve palsy grade IV post debulking of $\mathrm{PN}$ (6).

\section{Conclusion}

We declare that stridor symptoms in infants warrant early management via endoscopic assessments to assist early diagnosis. In our patient, a tracheostomy was done to secure the airway as PN had a mass effect on the airway. Major debulking was not done as it could cause more morbidity to the patient and the PN was a well-known benign slow growing tumor. Yearly MRI surveillance was deemed the best option for the patient.

Informed Consent: The mother of the patient provided the informed consent for the writing and publication of her child's case.

Peer-review: Externally peer-reviewed.

\section{Authorship contributions}

Conception: G.B.S., S.S.M.R., Design: G.B.S., S.S.M.R., Supervision: G.B.S., Data Collection and/or Processing: G.B.S., F.M.Z., S.H.M.P., S.S.M.R., M.F.A., Analysis and/or Interpretation: G.B.S., F.M.Z., S.H.M.P., S.S.M.P., M.F.A., Literature Review: F.M.Z., S.H.M.P., S.S.M.R., Writing: G.B.S., Critical Review: G.B.S., F.M.Z., S.H.M.P., S.S.M.R.

Conflict of Interest: No conflict interests declared by the authors. 
Financial Disclosure: This case report received no specific grant from any funding agency in the public, commercial, or not-for-profit sector.

\section{Main Points}

- Persistent stridor in a child need full endoscopic assessment.

- Plexiform Neurofibroma is an uncommon tumor but may present with common symptoms of stridor.

- In a patient with café-au-lait spots and family history of Neurofibromatosis Type-1, it would be helpful to assess head and tumor which is associated with Neurofibromatosis.

- Stridor with neck swelling require imaging prior to endoscopic assessment as it may guide us for the site of biopsy.

- Treatment of plexiform neurofibromatosis is challenging as it may cause more morbidity in a child.

- Total resection of invasive plexiform neurofibroma may be delayed as it is a slow growing tumor but require surveillance.

\section{References}

1. Sehgal VN, Srivastava G, Aggarwal AK, Oberai R. Plexiform neurofibromas in neurofibromatosis type 1 . Int J Dermatol 2009; 48: 971-4. [Crossref]

2. Joshi A, Lancelot M, Bhattacharjee NR, Polavarapu S, Beltaos E, Sobowale B. Extensive plexiform neurofibroma in a premature neonate. Clin Med Res 2015; 13; 36-40. [Crossref]

3. Pemov A, Li H, Patidar R, Hansen NF, Sindiri S, Hartley SW, et al. The primacy of NF1 loss as the driver of tumorigenesis in neurofibromatosis type 1-associated plexiform neurofibromas. Oncogene 2017; 36: 3168-77. [Crossref]

4. Mat C, Suhairi K, Hafizi M A, Hashim HZ, Nor NM, Mohamad I. Posterior neck gigantic plexiform neurofibromatosis. IJHHS 2018; 2: 236-48. [Crossref]

5. Asha'ari, ZA. Surgery for massive paediatric head and neck neurofibroma: two case reports. Int Med J Malays 2012; 11: 54-57. [Crossref]

6. Marocchio LS, Oliveira DT, Pereira MC, Soares CT, Fleury RN. Sporadic and multiple neurofibromas in the head and neck region: a retrospective study of 33 years. Clin Oral Investig 2007; 11: 1659. [Crossref]

7. Lum SG, Noor Liza I, Priatharisiny V, Saraiza A B, Goh BS Aetiological profile of paediatric stridor in a Malaysian tertiary hospital. Malays Fam Physician 2016; 11: 2-6. [Crossref]

8. Poussaint TY, Jaramillo D, Chang Y, Korf B. Interobserver reproducibility of volumetric MR imaging measurements of plexiform neurofibromas. AJR Am J Roentgenol 2003; 180: 41923. [Crossref]

9. Ransom ER, Yoon C, Manolidis S. Single stage near total resection of massive pediatric head and neck plexiform neurofibromas. Int J Pediatr Otorhinolaryngol 2006; 70: 1055-61. [Crossref]

10. Needle MN, Cnaan A, Dattilo J, Chatten J, Phillips PC, Shochat $\mathrm{S}$, et al. Prognostic signs in the surgical management of plexiform neurofibroma: the Children's Hospital of Philadelphia experience, 1974-1994. J Pediatr 1997; 131: 678-82. [Crossref] 